\title{
LA PALEONTOLOGÍA EN ARGENTINA ENTRE 1908 Y 1912. UNA MIRADA DIFERENTE DESDE MAR DEL PLATA
}

\author{
ALDO R. PRIETO
}

Instituto de Investigaciones Marinas y Costeras, CONICET-Universidad Nacional de Mar del Plata, Laboratorio de Paleoecología y Palinología, Funes 3250,7600 Mar del Plata, Argentina.

Recibido: 20 de Diciembre de 2015 - Aceptado: 26 de Mayo de 2016

Para citar este artículo: Aldo R. Prieto (2016). La paleontología en Argentina entre 1908 Y 1912. Una mirada diferente desde Mar del Plata. Publicación Electrónica de la Asociación Paleontológica Argentina 16 (2): $64-75$.

Link a este artículo: http://dx.doi.org/10.5710/PEAPA.26.05.2016.109

DESPLAZARSE HACIA ABAJO PARA ACCEDER AL ARTÍCULO

Asociación Paleontológica Argentina Maipú $6451^{\circ}$ piso, C1006ACG, Buenos Aires República Argentina Tel/Fax (54-11) 4326-7563 Web: www.apaleontologica.org.ar

Otros artículos en Publicación Electrónica de la APA 16(1):

\section{Garralla et al.}

RELACIONES PALEOFLORISTICAS DEL MIOCENO-PLIOCENO DEL NORTE ARGENTINO.

\section{Quintana}

TAFONOMÍA DE LOS MICROVERTEBRADOS DEL SITIO ARQUEOLÓGICO CUEVA TIXI (PLEISTOCENO TARDIOO-HOLOCENO TARDÍO), TANDILIA ORIENTAL.

\section{Zucol et al.}

CARACTERIZACIÓN FITOLÍTICA DE LA FORMACIÓN ARROYO FELICIANO (PLEISTOCENO SUPERIOR) EN LA LOCALIDAD PASO DUARTE, ENTRE RÍOS, ARGENTINA. 


\title{
LA PALEONTOLOGÍA EN ARGENTINA ENTRE 1908 Y 1912. UNA MIRADA DIFERENTE DESDE MAR DEL PLATA
}

\author{
ALDO R. PRIETO
}

Instituto de Investigaciones Marinas y Costeras, CONICET-Universidad Nacional de Mar del Plata, Laboratorio de Paleoecología y Palinología, Funes 3250,7600 Mar del Plata, Argentina. aprieto@mdp.edu.ar

\begin{abstract}
Resumen. Se analizan el panorama paleontológico en Argentina y el rol que tuvieron las políticas estatales y privadas en los ámbitos donde se desarrollaba la investigación paleontológica en relación con las políticas sociales entre 1908 y 1912. Este período se extiende desde la propuesta de construcción hasta la inauguración oficial del Asilo Saturnino E. Unzué en Mar del Plata. Se utilizan como ejemplo y se comparan dos instituciones estatales, el Museo Nacional de Buenos Aires y la Sociedad de Beneficencia de la Capital y sus protagonistas que convergieron en la difusa frontera entre lo público y lo privado, para analizar el rol del Estado en relación con la Paleontología y con la asistencia pública. Se discuten las diferencias entre "beneficencia" y "filantropía" en comparación con lo que ocurría en Estados Unidos de América durante ese periodo. El Asilo Unzué se presenta como un ejemplo del interés de las clases altas de Argentina en realizar obras de beneficencia, en lugar de suplementar los fondos del erario público para la construcción de museos o de ampliar las colecciones paleontológicas. Durante el período 1908-1912 los únicos representantes argentinos en las disciplinas paleontológicas fueron Florentino Ameghino y Francisco P. Moreno. Este período significó el final de la Paleontología como se había desarrollado desde la década de 1880. La ausencia de discípulos en todas las disciplinas paleontológicas y la prematura muerte de Ameghino conspiraron para que recién bien entrado el siglo XX se iniciara una etapa floreciente en la Paleontología.
\end{abstract}

Palabras clave. Paleontología. Museo Nacional de Buenos Aires. Sociedad de Beneficencia de la Capital. Asilo Unzué. Florentino Ameghino. Argentina.

Abstract. PALEONTOLOGY IN ARGENTINA BETWEEN 1908 AND 1912. A DIFFERENT POINT OF VIEW FROM MAR DEL PLATA. The paleontological framework and the role of the government and private policies in the area, where paleontological investigations were developed, are examined in relation to the social policies carried out in Argentina between 1908 and 1912. This period extends from the construction proposal until the official inauguration of the Asylum Saturnino E. Unzué in Mar del Plata. Two government institutions and their protagonists -the National Museum of Buenos Aires and the Charitable Society of the Capital- which converged on the fuzzy boundary between the public and private sectors, are used as examples and compared to analyze the role of the State in relation to Paleontology and the social policy. The difference between "charity" and "philanthropy" compared with what happened in the USA at that time is also discussed. The Asylum Unzué is presented as an example of how the patronage of the upper classes in Argentina was oriented toward charity works instead of supplementing the public treasury funds to build museums or enlarging paleontological collections. During the period from 1908 to 1912 , Florentino Ameghino and Francisco P. Moreno were the only Argentine representatives of the paleontological disciplines. This period marked the end of Paleontology as it had developed since 1880 in Argentina. The absence of disciples in all paleontological disciplines together with the premature death of Ameghino erased the field from Argentine research until well into the twentieth century, when it began to flourish anew.

Key words. Paleontology. National Museum of Buenos Aires. Charitable Society of Buenos Aires. Asylum Unzué. Florentino Ameghino. Argentina.

La Asociación Paleontológica Argentina (APA) fue fundada el 25 de noviembre de 1955 y, para recordar este acontecimiento, estableció esa fecha como el "Día del Paleontólogo". En 2015 se celebró el sexagésimo aniversario con la realización de la Reunión de Comunicaciones (RCAPA 2015) en la ciudad de Mar del Plata. El azar quiso que la misma tuviera como sede un lugar emblemático para la ciudad, las instalaciones del antiguo Asilo Saturnino E. Unzué (de ahora en adelante Asilo), conocido actualmente como "Espacio Cultural Unzué" dependiente del Ministerio de Desarrollo Social de la Nación. Esta conjunción de hechos originó la idea de revisar el panorama paleontológico en Argentina y el rol que tuvieron las políticas estatales y privadas en los ámbitos donde se desarrollaba la investigación paleontológica en relación con las políticas sociales entre 1908 y 1912. Este período se extiende desde la propuesta de construcción del 
Asilo hasta su inauguración oficial.

Se utilizó la historia del Asilo durante ese lapso como representación del interés de la elite gobernante en realizar obras de beneficencia y a la Sociedad de Beneficencia de la Capital (SBC), con sede en Buenos Aires, como la institución operadora de la política social del Estado. En contraparte, como representante de las instituciones donde se desarroIlaban las investigaciones paleontológicas, que eran promovidas por los particulares pero que dependían del Estado, el análisis se focalizó en el Museo Nacional de Buenos Aires (MNBA), "el Museo del País", actual Museo Argentino de Ciencias Naturales "Bernardino Rivadavia". El MNBA y la SBC habían sido creados en 1823 por Bernardino Rivadavia, entonces ministro de Martín Rodríguez. El MNBA estuvo dirigido hasta 1911 por Florentino Ameghino, el paleontólogo con mayor reconocimiento durante el período analizado (Fig. 1). En ese mismo tiempo la SBC adquirió la personería jurídica y se transformó en una institución oficial dependiente del Ministerio de Relaciones Exteriores y Culto.

Por otra parte, los afloramientos geológicos y los fósiles y restos arqueológicos provenientes de los acantilados al sur y al norte de la ciudad de Mar del Plata atrajeron durante el período analizado a varios naturalistas, entre ellos a los hermanos Ameghino. Esos hallazgos promovieron una de las discusiones científicas más importantes de la época, "la antigüedad del hombre en el Plata" en un momento singular de la ciencia y de la sociedad en Argentina.
De esta manera, en este trabajo se enmarca la historia del Asilo en las características sociales, políticas y culturales de la ciudad de Mar del Plata entre 1908 y 1912 y se analiza el estado de las investigaciones paleontológicas y el papel que desempeñaron el MNBA y la SBC y sus protagonistas en ese tiempo, en que convergieron en la difusa frontera entre lo público y lo privado. En particular se considera el rol del Estado en relación con la Paleontología y con la asistencia pública y se discuten las diferencias entre "beneficencia" y "filantropía" en comparación con lo que ocurría en los Estados Unidos de América durante ese periodo.

\section{El Asilo Saturnino Enrique Unzué en la Mar del Plata de principios del siglo XX}

Reseñar la historia del Asilo entre 1908 y 1912 constituye el nexo necesario para conectar las políticas estatales y la ciudad de Mar del Plata y para contextualizar el desarroIlo histórico de las disciplinas paleontológicas en este lapso. En esa época la ciudad representaba el ideal social de la elite gobernante, integrada en parte por las socias de la SBC quienes concurrían habitualmente al balneario durante el verano, de allí la conexión con la construcción del Asilo en esta ciudad (Delgado, 2011). A comienzo del siglo XX se realizaron numerosas obras de arquitectura que modificaron significativamente el paisaje de la ciudad de Mar del Plata. Durante la denominada Belle Époque, la primitiva villa balnearia se había transformado totalmente y las construc-
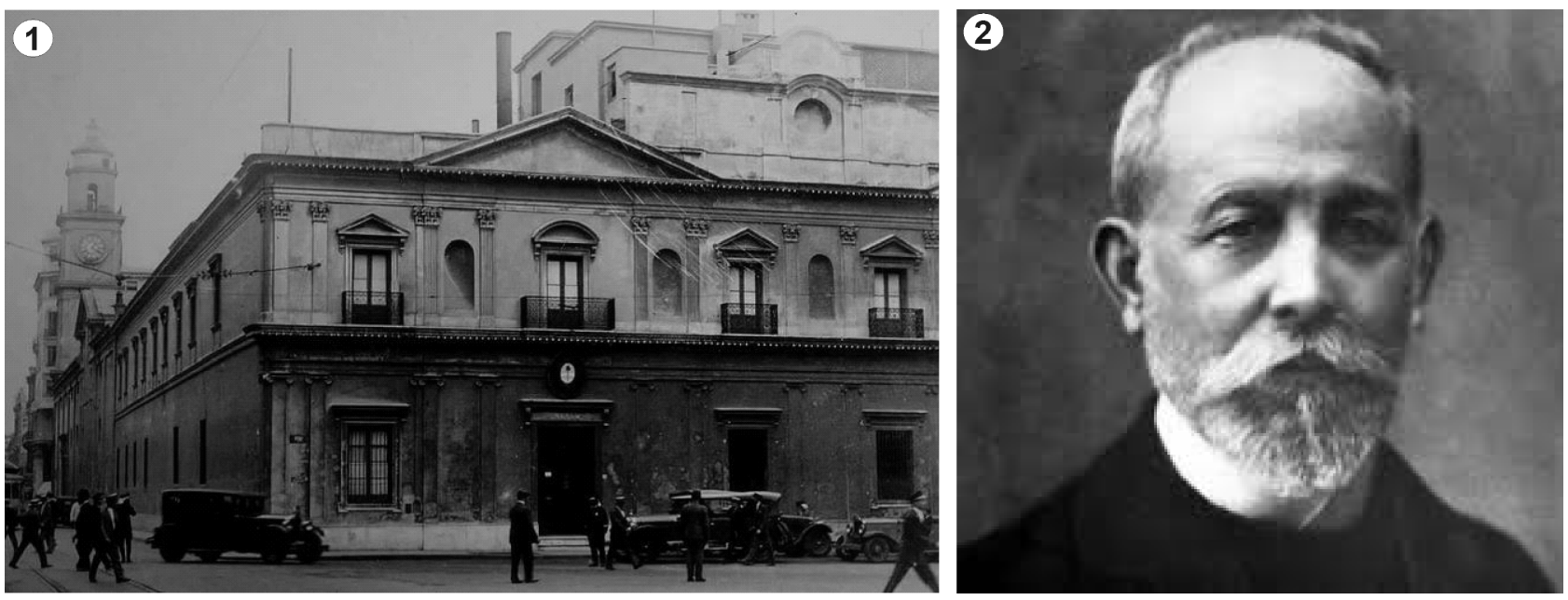

Figura 1. 1, Frente del Museo Nacional de Buenos Aires y la Universidad (1916). 2, Florentino Ameghino. 
ciones en la costa marplatense eran la representación de la riqueza de la elite agrícola-ganadera. El Bristol Hotel marcaba la estratificación social extrema entre los concurrentes habituales de la elite al balneario, durante su apogeo en 1910. En 1911 se determinó que la "Rambla de Lasalle", de madera, ya no estaba a la altura de los tiempos y se proyectó la primera rambla de mampostería, la "Rambla Bristol", conocida como Rambla Francesa, que se inauguró el 19 de enero de 1913. Hacia el interior del pueblo existían dos o tres núcleos poblacionales, donde vivían los obreros, casi todos inmigrantes, de la industria de la construcción (Bartolucci y Pilcic, 2009).

La transformación de la costa marplatense no solo estuvo marcada por la construcción de grandes residencias y hoteles, sino también por obras arquitectónicas relacionadas con la beneficencia, como el Asilo. La idea de construir el Asilo fue de María de los Remedios Unzué de Alvear (1861-1950), una de las tres marquesas pontificias de Argentina, junto a su hermana Concepción Natalia Unzué de Casares. La primera fue integrante de la SBC desde fines del siglo XIX, dos veces presidente y, posteriormente, socia honoraria hasta su fallecimiento. El 28 de junio de 1908, Dalmiro Iñiguez, en representación de María de los Remedios Unzué de Alvear, manifestó al Comisionado Municipal de Mar del Plata, Juan José Urdinarrain, "que la señora tiene resuelto construir en la mitad S.O. de la chacra 328, de su propiedad, un Asilo-Sanatorio destinado a niñas" (Expte. 278-B1908, iniciado por María Unzué de Alvear el 2/1/1908; nomenclatura catastral: VI-B-182I-1). Cumplido este trá- mite, las hermanas Unzué encomendaron al arquitecto francés Paul-Louis Faure-Dujarric el proyecto de un edificio "destinado al alojamiento y educación de niñas pobres" (Delgado, 2001, p. 92) y la construcción de un Oratorio en su planta central y le recomendaron al profesional la utilización de los más nobles materiales de la época. Faure-Dujarric proyectó un edificio que por sus propias características representó para la época lo más adelantado desde el punto de vista arquitectónico y por su aspecto comunicativo constituyó la más extrema vanguardia de las cuestiones estéticas en la disciplina (Somma, 2013). En 1909 se inició la construcción estructural del edificio bajo la dirección del constructor de Mar del Plata, Mauricio Cremonte secundado por el francés León Fragnaud, siguiendo minuciosamente las instrucciones del proyectista, de tal manera que encajaran perfectamente los embaldosados, los revestimientos de muros, los fustes de columnas, los capiteles, las bases, la orfebrería, los herrajes y la carpintería del interior que se estaban realizando en los talleres de Curzio Caponetti-Esegui en Roma. Las luminarias y los candelabros fueron obra de artesanos franceses.

Arquitectónicamente, es un edificio de pabellones, de planta en $\mathrm{H}$, de estilo ecléctico con influencia de las vanguardias modernas de principios del siglo XX, en especial de la contemporánea Secesión Vienesa, movimiento artísticoarquitectónico dentro del academicismo, también conocido como modernismo (Fig. 2). En marzo de 1910 las hermanas Unzué comunicaron al Papa Pío X que "han hecho edificar una gran Capilla dedicada a la Inmaculada Concepción con un Asilo
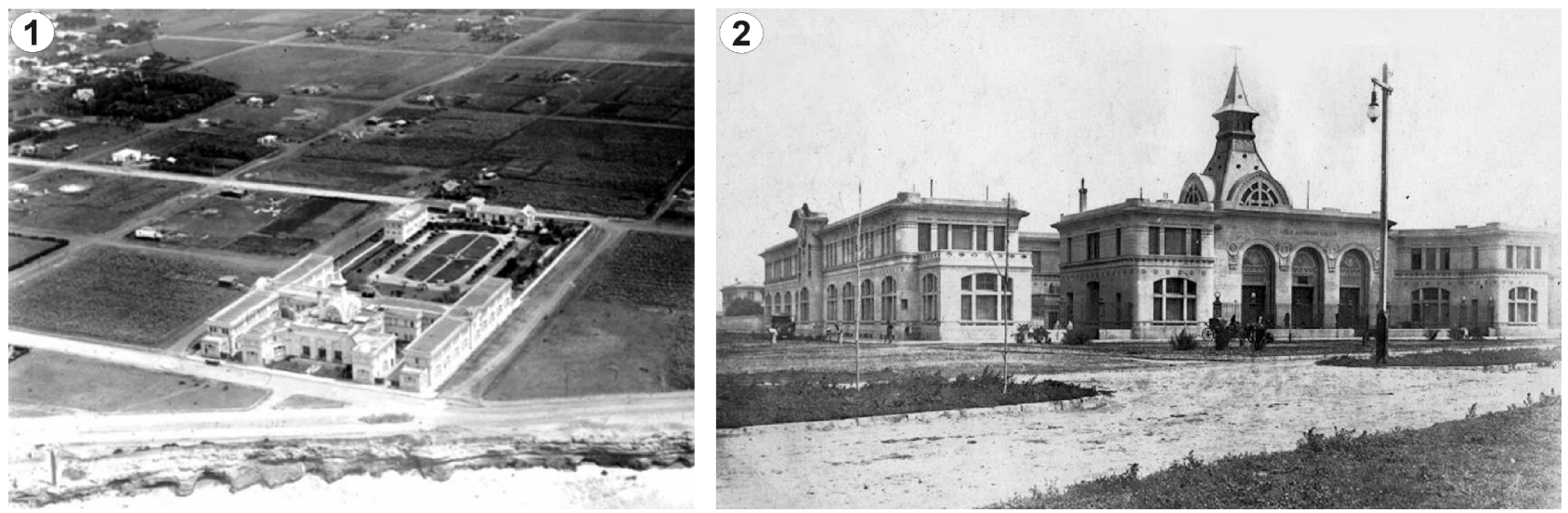

Figura 2. 1, Foto aérea del Asilo Saturnino E. Unzué, tomada por Bruno Bernardo Gelber ca. 1924/25. 2, Frente del Asilo visto desde el mar (ca. 1920). 
anejo [...] donde se podrán recibir 300 niñas pobres y el qual [sic] confiado a una Congregación religiosa" (en carta original) y solicitaron " una Indulgencia Plenaria" (en carta original). La respuesta favorable de Pío X llegó en el día de la Inmaculada Concepción, en original y al pie de la carta enviada por las hermanas Unzué. Por tal motivo, el 8 de diciembre de 1910 el Oratorio de estilo neobizantino, único en América, fue consagrado a la advocación de la Inmaculada Concepción de María Virgen. El 7 de septiembre de 1911, las hermanas Unzué donaron a la SBC en la persona de su presidente, Sofía Arning de Bengolea, el inmueble, las tierras, las dependencias y las instalaciones, la donación particular más importante que recibió esta institución desde su fundación (Sociedad de Beneficencia de la Capital, 1913, p. 63). Ese mismo año llegaron las Hermanas Franciscanas Misioneras del Corazón de María, encargadas de la atención del Asilo. El 5 de marzo de 1912, con la presencia de Roque Sáenz Peña, entonces presidente de la Nación y de autoridades municipales, provinciales y nacionales, se inauguró oficialmente el Asilo con el nombre de "Saturnino Enrique Unzué" en honor al padre de las donantes (Ayeza, 1995). El edificio se transformó en un hito de referencia de la costa marplatense, no solo por el emplazamiento frente al mar, por su tamaño y por su uso, sino, fundamentalmente por sus particularidades formales (Somma, 2013). Para 1915 figuraba en las cartas náuticas de la costa de la provincia de Buenos Aires, no solo indicado, sino también ilustrado, junto con los otros hitos distinguibles desde el mar: el Faro Mogotes, Ia Iglesia San Pedro (actual Catedral de los Santos Pedro y Cecilia) y el Club Mar del Plata (derrumbado en 1961) (Servicio Hidrográfico del Ministerio de Marina, 1916). El valor simbólico del emplazamiento continúa siendo dominante y un siglo después de su construcción permanece como tal en el paisaje urbano costero de la ciudad de Mar del Plata.

\section{La Sociedad de Beneficencia en la época de la construcción del Asilo}

El género femenino caracterizaba la vida de la SBC que estaba integrada por las esposas y por las parientas más próximas de los grandes terratenientes, comerciantes y propietarios de la ciudad y de la provincia de Buenos Aires. Esta posición social les aseguraba una cercanía al poder que explica mucho del éxito de la gestión de la SBC. Esta institución se hacía cargo en forma privada, pero en gran parte con fondos públicos, de las principales tareas de beneficencia y de asistencia social hasta 1908, cuando se transformó en una institución oficial. Estas damas tenían acceso libre a los despachos oficiales y mantenían un estrecho vínculo con diputados y senadores, con quienes compartían reuniones sociales y culturales (Golbert y Roca, 2010). De esta manera, tenían una activa participación en la vida pública y también trabajaban como asistentes sociales, tenían poder y reconocimiento para introducirse en los sectores marginales (Delgado, 2011) y formaban parte de la elite que realizaba donaciones, como lo hicieron muchos de los integrantes de la familia Unzué para la ciudad de Mar del Plata (Delgado, 2011, p. 55). La SBC tenía a su cargo administrar y adjudicar una porción del dinero que provenía en una alta proporción del Presupuesto Nacional, ya que gozaba de autonomía para administrar los fondos del Estado. Recibía también dinero de lo recaudado por la Lotería de Beneficencia Nacional y de las donaciones privadas que eran destinadas en su totalidad a obras de beneficencia. Aunque el aporte privado fue, en términos comparativos y a lo largo de una serie histórica 1823-1942, notablemente inferior al público, no puede decirse que fuese insignificante. Incluso en algunos años, como en 1907 y en 1911, los aportes privados fueron mayores que los del Estado, considerando solamente las donaciones recibidas en efectivos y no las tierras, campos y otros inmuebles, como por ejemplo el Asilo Unzué (Thompson y Campetella, 1994). "La caridad privada se ha manifestado el año ppdo [1911] excepcionalmente favorable, elevándose el importe total de su contribución por sobre todos los resultados anuales del mismo concepto, conocidos en la Sociedad" (Sociedad de Beneficencia de la Capital, 1913, p. 10). Este fue el rol histórico que les correspondió a las mujeres de la elite en la SBC y cuya acción impactó directamente en la conformación de una identidad nacional (Delgado, 2011).

\section{Las investigaciones paleontológicas entre 1908 y 1912}

Diversos trabajos sobre el desarrollo de la Paleontología en Argentina (Pascual, 1961; Reig, 1961; Stipanicic, 1971; Archangelsky, 1981; Riccardi, 1981, 2005, 2011; Ottone, 2005) constituyeron la base para realizar la reseña de las investigaciones paleontológicas y sus protagonistas entre 1908 y 1912. En esas contribuciones este período fue in- 
cluido sin distinción dentro de la transición de fin del siglo XIX a principios del siglo XX y sin consideraciones particulares.

Según Pascual (1961), las investigaciones paleontológicas durante el lapso analizado podrían enmarcarse en tres períodos del desarrollo científico de Argentina. Uno está asociado con el de un país de paso, que se inició en las primeras décadas del siglo XIX, donde hombres y expediciones científicas extranjeras efectuaban observaciones y realizaban colecciones que enriquecían sus museos y los resultados se publicaban en sus propios países y solo resultaban beneficiosos de manera indirecta para la Argentina. El otro, que se inició con posterioridad a la mitad del siglo XIX está asociado con el establecimiento en el país de científicos extranjeros, porque las autoridades entendieron que su influencia, su experiencia y su enseñanza permitirían formar discípulos y serían beneficiosas para el desarrollo científico del país. El tercero es aquel cuando "la paleontología argentina obtiene su carta de ciudadanía" (Pascual, 1961, p. 88) y se inició con la actividad creadora de F. Ameghino y la labor de campo de su hermano Carlos (Reig, 1961).

Para el período, quien estuvo más estrechamente vinculado a la Paleobotánica, fue el sueco Thore Gustaf Halle (Fig. 3.1), no solo por la importancia de sus trabajos sino también por su presencia física en la región austral de Argentina (Archangelsky, 1981). Integró la expedición dirigida por el botánico Carl Skottsberg entre 1907 y 1909 por América del Sur (Islas Malvinas y parte continental de Argentina, Chile y Brasil). El material recolectado por Halle durante esa expedición, junto con las plantas fósiles del Mesozoico de la Tierra de Graham (Península Antártica Argentina), recogidas por Johan Gunnar Andersson en la expedición de Nordenskjöld al Polo Sur (1901-1903) fueron motivo de varias publicaciones que aparecieron con posterioridad a 1912. En 1911, Halle publicó On the geological structure and history of the Falkland Islands, que fue su Tesis Doctoral, donde se destaca el descubrimiento de la Flora de Glossopteris. Otros investigadores que estudiaron y publicaron materiales de plantas fósiles provenientes de diferentes localidades de Argentina entre 1908 y 1912 fueron Walther Gothan y Per Karl Hjalmar Dusen. Para el período, es importante la mención de Fritz [Federico] Kurtz (Fig. 3.2), botánico alemán que se radicó en Córdoba en 1884 donde se hizo cargo de la cátedra de Botánica de la Universidad Nacional de Córdoba, en la que permaneció hasta1915. Fue director del Museo de Botánica y como miembro activo de la Academia Nacional de Ciencias de Córdoba, mantuvo un intercambio fluido de ideas y de materiales con científicos y con instituciones de todo el mundo. Se dedicó a describir los materiales de plantas fósiles coleccionados por varios naturalistas, aunque gran parte de su obra sobre paleobotánica fue publicada después de su fallecimiento, ocurrido en 1920 (Stipanicic, 1971; Ottone, 2005). A pesar del período prolongado que Kurtz permaneció al frente de la Cátedra de Botánica, no logró formar discípulos que continuaran sus estudios. Las obras de Halle y Kurtz marcan la superposición de dos de los períodos en el desarrollo científico señalados al inicio para el lapso 1908-1912.

Los trabajos sobre invertebrados para el período 19081912 incluyeron la mayor parte de las monografías sobre las megafaunas del Fanerozoico (Riccardi, 2005). Muchos de esos trabajos se realizaron en el marco de expediciones organizadas entre fines del siglo XIX e inicios del siglo XX por investigadores de instituciones extranjeras como las de Nordenskjöld, C. Skottsberg y John Bell Hatcher a la Patagonia austral y la de Gustav Steinmann al oeste de América del Sur entre 1882 y 1908, con excepción de aquellas realizadas por científicos del Museo de La Plata, asociadas con el viaje de Rodolfo Hauthal en 1898 y en 1900 y de la Academia Nacional de Ciencias de Córdoba, a las que se deben las colecciones que efectuó Guillermo Bodenbender. Parte de estos materiales fueron publicados en 1908 por F. Favre y W. Paulcke en el exterior (Riccardi, 2011).

Los principales trabajos sobre invertebrados fósiles fueron publicados con anterioridad a 1908 por el malacólogo alemán-brasileño Hermann von Ihering (Fig. 3.3), coleccionados por Roth y Hauthal y con posterioridad a 1910 por el argentino Martín Doello Jurado. Von Ihering, un aliado de F. Ameghino, complementó los estudios sobre vertebrados fósiles realizados por los hermanos Ameghino desde el MNBA (Riccardi, 1981) y determinó los moluscos que F. Ameghino había recolectado en sus tres viajes a Mar del Plata en 1908 (Ameghino, 1908, pp. 375, 376, 388, 389, 393, 407, 408). Santiago Roth, quien era el encargado de la sección Paleontología del Museo de La Plata, contribuyó con su trabajo a incrementar y ordenar las colecciones de invertebrados, e 

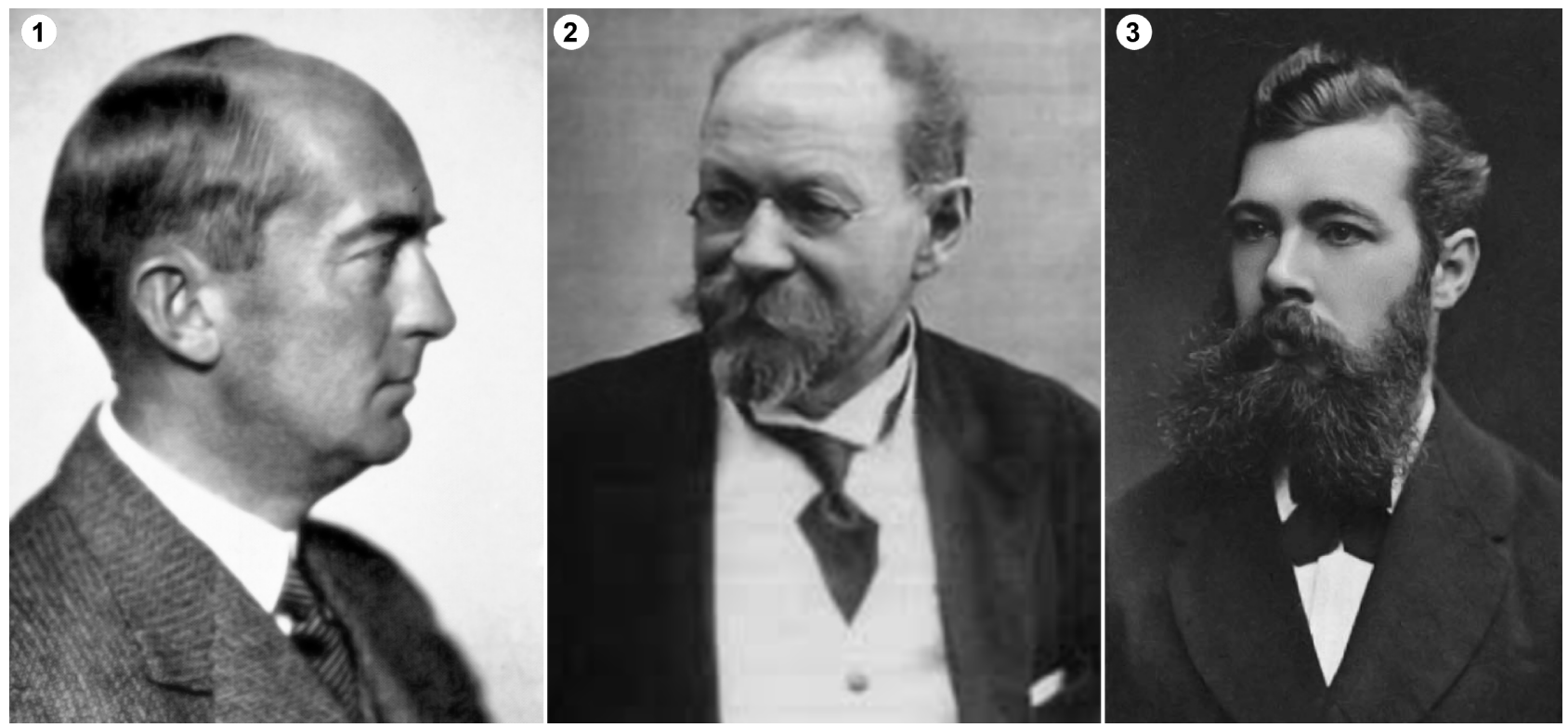

Figura 3. 1, Thore Gustaf Halle. 2, Fritz [Federico] Kurtz. 3, Hermann von Ihering.

incluso descubrió un yacimiento de importancia. Sin embargo, sus estudios se orientaron hacia los vertebrados fósiles (Riccardi, 2011), aunque fue "más geólogo que paleontólogo" (Pascual, 1961, p. 90). Los estudios de invertebrados fósiles, mayormente dirigidos al conocimiento de las faunas y a sus vinculaciones además de documentar la existencia de terrenos de edades determinadas (Riccardi, 1981), al igual que los de paleobotánica, fueron realizados en su mayoría por extranjeros durante el lapso 1908-1912.

El impulso de la Paleontología se debió al estudio de los vertebrados fósiles más que a los de invertebrados y floras fósiles. La paleontología de los mamíferos concentró la atención de la mayoría de los naturalistas locales y de numerosos paleontólogos extranjeros hasta la primera década del siglo XX (Vizcaíno et al., 2013). Hacia 1907, este interés decayó cuando la posibilidad del hombre terciario comenzó a ser agitada, entre otros, por F. Ameghino (Podgorny, 2001, 2009). Los estudios sobre los vertebrados fósiles alcanzaron personalidad propia y relieves destacados en Argentina con los trabajos de F. Ameghino. Otros paleontólogos contemporáneos que se ocuparon de los mamíferos fósiles fueron Francisco Pascasio Moreno (argentino), Alcides Mercerat (francés/suizo) y Santiago Roth (suizo). Este último, desde el Museo de La Plata, contribuyó a propagar la idea de que F. Ameghino fraguaba las evidencias (Podgorny,
2009). Sin embargo, la obra de todos ellos ha quedado un tanto desdibujada ante el monumental aporte cuantitativo y cualitativo de la obra de F. Ameghino (Pascual, 1961; Reig, 1961). Antes de finalizar el período analizado, este había muerto sin dejar discípulos que lo continuaran, pero, paradójicamente, hizo del MNBA un centro para los estudios de la paleontología de vertebrados (Asúa, 2012).

Si bien la investigación en Paleontología muestra una cierta continuidad entre fines del siglo XIX y principios del siglo XX, la investigación de esta rama de la ciencia experimentó una cierta declinación después de 1890. Durante el lapso 1908-1912 hubo una superposición de los tres períodos del desarrollo científico de Argentina en las investigaciones en Paleontología y es notable la ausencia de discípulos en todas las disciplinas paleontológicas.

\section{Los científicos relacionados con la paleontología que visi- taron Mar del Plata y los afloramientos próximos entre 1908 y 1912}

Florentino Ameghino visitó Mar del Plata y los afloramientos próximos entre 1908 y 1910 y lo hizo en cuatro oportunidades. En 1908 realizó "[... ] tres viajes á Mar del Plata y á la región que se extiende hacia el sur hasta más allá de la Barranca de los Lobos, recorriendo á pie la costa atlántica, desde la boca de la Mar Chiquita al norte hasta la boca del 
arroyo Chocorí al sur" (Ameghino, 1908, p. 344). Realizó los dos primeros viajes acompañado por su esposa, Leontina Poirier y el tercero junto a su hermano Carlos, en carácter de naturalista viajero, y al preparador Antonio Pozzi, ambos empleados del MNBA. Se alojaron en la Estancia "Chapalmalán" de Miguel Alfredo Martínez de Hoz "[...] propietario de la mayor parte de esa región y ciertamente uno de los hacendados más progresistas de la provincia de Buenos Aires" (Ameghino, 1908, p. 351). Estuvo en la playa Bristol e hizo observaciones "en el mismo balneario á solo unos 200 metros de la rambla [...]" (Ameghino, 1908, p. 393) y señaló que "con los trabajos de transformación de la playa dentro de poco [el depósito que estaba siguiendo] habrá completamente desaparecido" (Ameghino, 1908, pp. 396, 398). También visitó los acantilados de la zona norte donde se construyó el Asilo "[...] los que en Mar del Plata llaman las 'Barrancas del Norte'" (Ameghino, 1908, p. 398) y aconsejó que las visiten para que se den "cuenta del error en que están [...] todos aquellos que están acostumbrados á considerar la formación pampeana como un depósito de limo uniforme..." (Ameghino, 1908, p. 401). Si bien no se alojó en el Bristol Hotel estuvo allí "con el mecánico del establecimiento" quien le brindó datos sobre la profundidad y los tipos de sedimentos de una perforación "reciente" de 83 m de profundidad que se había realizado en el hotel para el agua potable (Ameghino, 1908, pp. 397, 405). Este dato contradice la versión de Ayeza (1995) de que los primeros pozos se perforaron en el Asilo en 1909.

Estos tres viajes tuvieron como objetivo esclarecer si las capas que afloraban en las barrancas al sur de Mar del Plata pertenecían al pampeano inferior o tenía una edad semejante a las capas de Monte Hermoso, que serían "de una época geológica muy anterior" (Ameghino, 1908, p. 421). Estableció que "representan un nuevo horizonte un poco más reciente" que el de las capas de Monte Hermoso, y las designó con el nombre de "chapalmalense" además de reconocer "una fauna de mamíferos completamente nueva" que utilizó para probar que se trataba de "un nuevo horizonte distinto del hermosense" y para indicar en primer término "la existencia del hombre ó de su precursor [...]" en el chapalmalense (Ameghino, 1908, p. 421). La publicación de la descripción detallada de esta fauna en una memoria especial del Museo de La Plata como tenía pensado nunca ocurrió.
El 24 de mayo de 1910 el antropólogo checo-norteamericano Aleš Hrdlička y el geólogo Bailey Willis (Fig. 4) viajaron desde Buenos Aires a Mar del Plata, en misión del Smithsonian Institution, para unirse a los hermanos Ameghino "a few days after" (unos pocos días después) para realizar "examinations [...] of the coast from north of Mar del Plata to the Barrancas de los Lobos [...], a section very important from the standpoint of archeology and geology; of the more inland Laguna de los Padres, the neighborhood of which was occupied up to late historic times by a small body of Indians; of the coast about and to the northeast of Miramar, a region interesting archeologically, geologically, and because of finds, slightly farther to the south, of two "fossil" human crania [...]" (exámenes....de la costa desde el norte de Mar del Plata hasta las Barrancas de los Lobos..., una sección muy importante desde el punto de vista de la arqueología y la geología; de la más interior Laguna de los Padres, los alrededores de la cual fue ocupada hasta antes de los tiempos históricos por un pequeño grupo de Indios; de la costa cerca y al noreste de Miramar, una región arqueológicamente y geológicamente interesante, y debido a los hallazgos, un poco más al sur, de dos cráneos humanos "fósiles") (Hrdlička, 1912, prólogo p. VII). Luego continuaron viaje al Arroyo del Moro (sur de Miramar), a Necochea y a otros lugares de la costa hasta Monte Hermoso y sin la compañía de los hermanos Ameghino hasta Río Negro.

Posiblemente hicieron el trayecto desde Buenos Aires hasta Mar del Plata en tren y luego en galera hasta Miramar, ya que el tren recién llegó a esta localidad en febrero de 1911. No hay datos sobre dónde se hospedaron Hrdlička y Willis en Mar del Plata. En Miramar probablemente se alojaron en el Hotel Argentino, que se hallaba ubicado en la actual Peatonal 9 de julio esquina 20 y que era el mejor de esa época. A pesar de que F. Ameghino le había advertido a Hrdlička que "L'époque la plus propice, du moins en vue de faire des excursions, est de Septembre à Novembre, et de Mars á Mai" (La época más propicia, al menos para hacer excursiones es de septiembre a noviembre y de marzo a mayo) (Torcelli, 1936; carta 2495) este decidió viajar a finales de mayo, por lo que resulta casi obvio que se quejara del frío en la zona y en los hoteles (Podgorny y Politis, 2000). Le reconoce a F. Ameghino y a su hermano el esfuerzo que hicieron para acompañarlos "[...] notwithstanding the inclement 
season, for nearly three weeks [...]" (a pesar de la temporada inclemente, de casi tres semanas) (Hrdlička, 1912, prólogo
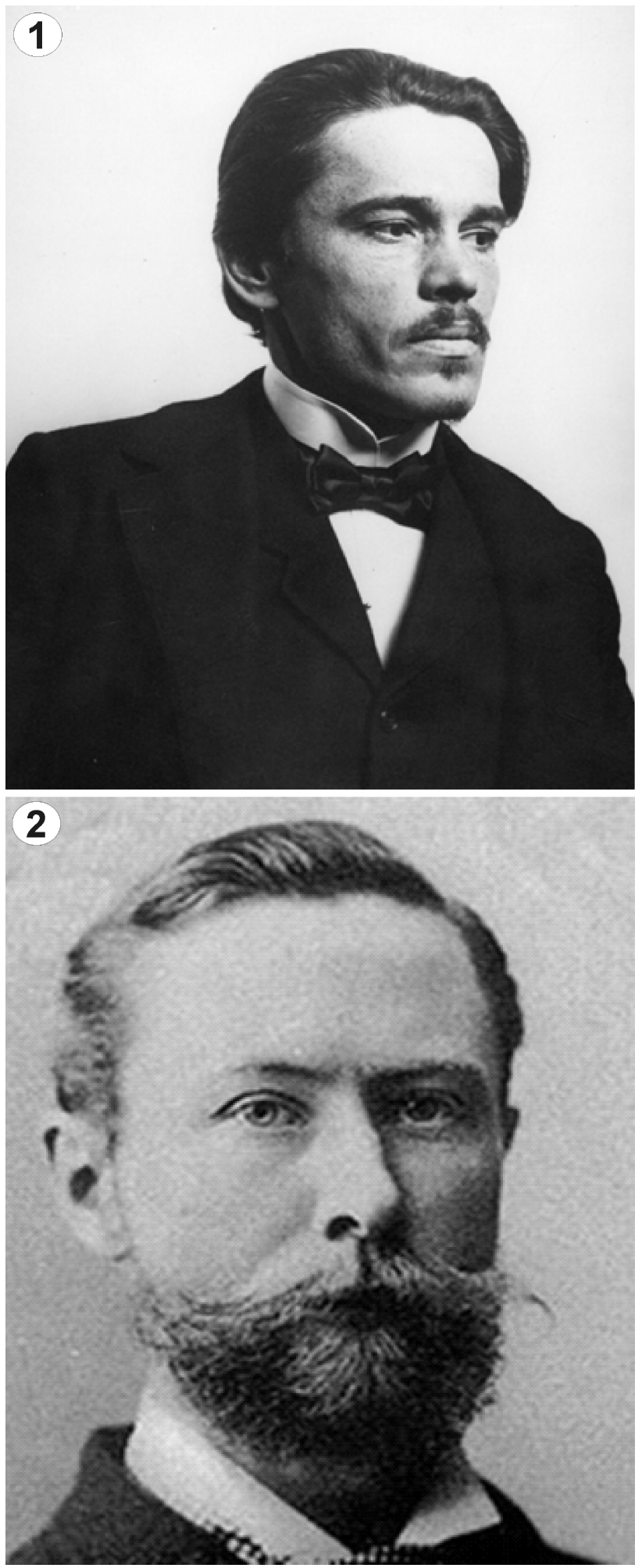

Figura 4. 1, Aleš Hrdlička. 2, Bailey Willis. p. VII). Este último viaje de F. Ameghino tuvo como objetivo acompañar a los científicos del Smithsonian Institution "[...] from point to point along the coast where vestiges of ancient man or his forerunners were believed to have been discovered [...]" (de punto a punto a lo largo de la costa donde se cree que los vestigios del hombre antiguo o sus precursores han sido descubiertos) (Hrdlička, 1912, prólogo p. VII). Los resultados de este viaje, contrarios a las hipótesis de F. Ameghino, fueron publicaros en 1912 por Hrdlička, cuando F. Ameghino ya había fallecido, y determinaron un punto de inflexión en el debate sobre la antigüedad del hombre en América, sobre todo a nivel internacional.

\section{El Museo Nacional de Buenos Aires entre 1908 y 1912}

Para el lapso 1908-1912, el Museo de la Plata, la Academia Nacional de Ciencias con sede en Córdoba y el MNBA, donde el género masculino protagonizaba el funcionamiento y las relaciones políticas e institucionales, formaban el conjunto de instituciones científicas de envergadura que establecieron los fundamentos del desarrollo que las ciencias paleontológicas tendrían posteriormente en la Argentina. En contraste con las dos primeras, el MNBA no disponía de un edificio adecuado y se encontraba en estado de ruina (Podgorny, 2009). Era un edificio desvencijado sobrecargado de piezas paleontológicas, amontonadas en sótanos y en entrepisos (Fig. 5); la falta de espacio y los peligros de derrumbe (Asúa, 2012) fueron vividos como una tortura por $F$. Ameghino (Podgorny, 2009). A pesar del interés que tenían los delegados extranjeros, que llegaron para el Congreso Científico Internacional Americano y el $17^{\circ}$ Congreso de Americanistas en 1910, en las colecciones y en los supuestos hallazgos del "precursor del hombre" depositados en el MNBA, el edificio y el estado de abarrotamiento de las colecciones, lo hicieron impresentable (Podgorny, 2009) y permaneció cerrado al público durante el Centenario como testimonio del descalabro (Asúa, 2012) y continuó clausurado al público hasta 1914.

F. Ameghino, como director del MNBA desde 1902, le dio un énfasis más paleontológico y científico que su antecesor, Burmeister. Cuando se propuso como posible director del MNBA señaló que el museo se beneficiaría con la incorporación de su colección paleontológica de casi 100.000 piezas (Asúa, 2012), hecho que no ocurrió hasta la década de 1930 


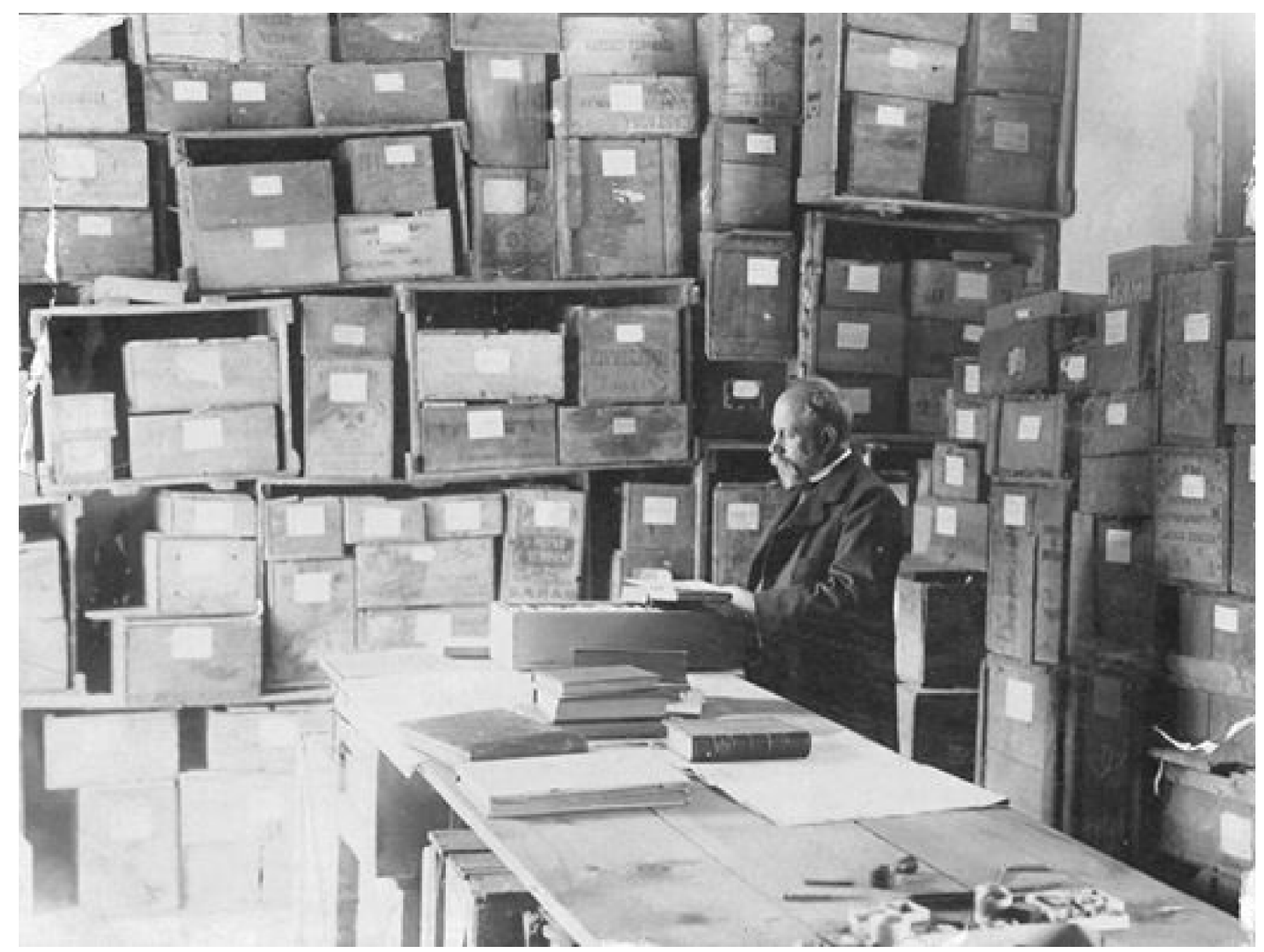

Figura 5. F. Ameghino en el Museo Nacional de Buenos Aires ca. 1910 (tomado de Podgorny, 2009).

cuando fueron adquiridas por las autoridades nacionales después del fallecimiento de los hermanos Ameghino (Fernicola, 2011). Esto muestra la importancia que tenían las colecciones paleontológicas privadas y que el comercio de fósiles entre particulares e instituciones era una práctica común durante el lapso analizado.

Todas las tratativas de trasladar o de ampliar el museo que realizó F. Ameghino no pudieron concretarse y recién bien entrado el siglo XX pudo construirse el edificio donde se sitúa actualmente. Según Podgorny (2009) todas las propuestas de obtención de fondos para ampliar y/o para modificar el edificio o incluso para construir uno nuevo estaban dirigidas al Estado. Los presupuestos que se presentaron para la construcción y/o ampliación entre 1903 y 1907 no superaban los $885.000 \mathrm{~m} \$ \mathrm{n}$. El contraste con el presupuesto disponible para el edificio del Asilo es notable. En ese tiempo se estaba construyendo en Mar del Plata un edificio de $8697 \mathrm{~m}^{2}$ bajo el mecenazgo privado de integrantes de la clase alta argentina que "han invertido generosamente una cantidad aproximada de $\$$ dos millones" [\$=m\$n] (Sociedad de Beneficencia de la Capital, 1913, p. 11). Aparentemente, existía una cierta incapacidad en F. Ameghino para negociar en el contexto cambiante de las alianzas políticas en el Buenos Aires de principios del siglo XX (Podgorny et al., 2015) y tal vez por eso no se recurría al mecenazgo privado de la clase alta, o bien no había interés de parte de esta en suplementar los fondos del erario público para este tipo de obras, a diferencia de lo que ocurría en los Estados Unidos con los ejemplos de J. Smithson, A. Carnegie, R. Colgate, J.P. Morgan, entre otros. Algunos de estos acaudalados banqueros e industriales filántropos, con sus ganancias siderales colaboraban tanto en la adquisición de nuevas colecciones paleontológicas como en la expansión de los edificios y cubrían desde sus fundaciones privadas los espacios dejados por las políticas gubernamentales. 


\section{La beneficencia y la filantropía durante el período 1908-1912}

La beneficencia y la filantropía constituirían conceptos diferentes pero que se imbrican con los sectores de poder en cada circunstancia (Delgado, 2011). Así, durante el lapso 1908-1912, la Iglesia y el Estado configuraron un estilo de acción que privilegió el rol femenino para el logro de las tareas políticas. La beneficencia en Argentina tenía una fuerte impronta católica, asociada con la "caridad cristiana", que principalmente trataba de aliviar los sufrimientos de los pobres. Esta beneficencia organizada en la SBC se transformó en política social al estar incorporada totalmente al sistema burocrático del Estado (Delgado, 2011). En este sentido podría interpretarse como filantropía, entendida esta como una respuesta humanitaria a las condiciones de los pobres institucionalmente canalizada (Sulek, 2010). En cambio, para ese tiempo la filantropía estaba asociada con "la filantropía científica" que pretendía abordar las causas fundamentales de la pobreza para lograr soluciones permanentes de la misma y a otros males sociales. Quienes postulan esta distinción a menudo apuntan a la influencia seminal de las entidades filantrópicas como la de Rockefeller o Carnegie. A diferencia de las clases adineradas de los Estados Unidos, que perpetuaban su nombre y su gloria en edificios muchas veces relacionados con los museos, las clases altas de Argentina lo hacían en edificios para asilos y hospitales, como el Asilo. También existen diferencias en la concepción edilicia entre los museos norteamericanos y argentinos (Podgorny, 2009). Los primeros eran edificios con salas modulares que permitían el crecimiento; en cambio, los museos argentinos se "concentraban en círculos de bello diseño y fácil circulación pero de muy difícil ampliación" (Podgorny, 2009, p. 219). El Museo de La Plata se transformó en un ejemplo a imitar ya que se constituyó en el digno representante de la identificación entre las colecciones y el medio social argentino. Además, en ese entonces las colecciones argentinas eran insignificantes comparadas con las grandes y colosales colecciones paleontológicas de los museos norteamericanos que se incrementaban gracias a los grandes capitales de ese país (Podgorny, 2009). El MNBA y SBC muestran el rol diferente del Estado en relación con la Paleontología y con la asistencia pública y la participación que tuvieron los diferentes protagonistas para alcanzar los objetivos insti- tucionales. Mientras los museos argentinos eran promovidos por particulares, pero creados con los vaivenes del Estado, en la asistencia pública, que también era dependiente del Estado, eran los integrantes de la clase alta argentina quienes promovían la construcción de asilos, de hospitales y de escuelas con su propio dinero y los donaban al Estado.

\section{A MODO DE CONCLUSIÓN}

Para el lapso 1908-1912 el MNBA y SBC fueron dos de las instituciones que muestran el rol diferente del Estado en relación con la Paleontología y con la asistencia pública. El Asilo, como un caso particular, es un ejemplo del interés de las clases altas en realizar obras de beneficencia directamente relacionadas con la educación y con la formación religiosa de las niñas "pobres" en particular y de las mujeres en general y no en suplementar los fondos del erario público para la construcción de museos o para ampliar las colecciones paleontológicas. Resulta clara la diferencia entre esta "beneficencia" y la "filantropía" de las clases adineradas de los Estados Unidos. Sin dudas la impronta católica, hispana y agroexportadora en las primeras marcó el contraste con una sociedad protestante, anglosajona e industrializada que representan las segundas.

Durante ese período, los únicos representantes argentinos en las disciplinas paleontológicas fueron F. Ameghino y F.P. Moreno. Como en el resto de las ciencias básicas en Argentina, la mayoría de la investigación paleontológica estaba en manos de científicos de los países de habla alemana. F. Ameghino tuvo el rol protagónico dentro de la Paleontología de la época, no solo por su reconocimiento a nivel nacional e internacional, sino también por su rol como director de MNBA. El género masculino protagonizaba el funcionamiento, las relaciones políticas e institucionales y las investigaciones paleontológicas mientras que el Estado había relegado al rol femenino la tarea de beneficencia. Sin embargo, como señala Delgado (2011), esta no fue meramente un pasatiempo, sino que fue la instancia a través de la cual las mujeres desempeñaron un papel cada vez más activo en la vida pública.

El período 1908-1912 significó el final de la Paleontología como se había desarrollado desde la década de 1880. Sin dudas, la prematura muerte de F. Ameghino y la ausencia de discípulos en todas las disciplinas paleontológicas 
conspiraron para que recién bien entrado el siglo XX se iniciara una etapa floreciente en la Paleontología. Esta se modularía durante las próximas décadas hasta alcanzar el camino de la profesionalización a partir de 1930, como ocurrió con otras ramas de las ciencias.

Para el lapso estudiado, la ciudad de Mar del Plata representó el ideal social de la elite gobernante, cuyos integrantes que concurrían al balneario durante el verano, dejaron una impronta perdurable en obras de beneficencia ejemplificada con el edificio del Asilo Saturnino E. Unzué. Por otro lado, los acantilados próximos a la ciudad también atrajeron a varios naturalistas, entre ellos a F. Ameghino, durante ese tiempo. Los datos geológicos y los fósiles y restos arqueológicos obtenidos de esos afloramientos dieron apoyo o rechazo a una de las discusiones científicas más importantes de la época, "la antigüedad del hombre en el Plata".

\section{AGRADECIMIENTOS}

A M. de Asúa por sus sugerencias, sus consejos y la lectura crítica de la primera versión del manuscrito. Quiero reconocer la ayuda de las bibliotecarias del INIDEP y de la Biblioteca Nacional y de las autoridades del Espacio Cultural Unzué. A M.S. Bargo, M. Bond, D. Boh, M. Bonomo, M. Checotti, C. Deschamps, F. I. Isla, I. Podgorny, C. Quintana, V. Recanatesi y M.E. Millares por sus desinteresadas colaboraciones con bibliografía y con documentos. A J.L. Cionchi por aclararme el tema de las perforaciones para obtener agua subterránea en Mar del Plata. A J.C. Fernicola y A. Kramarz por los oportunos comentarios realizados durante la presentación de una parte de este trabajo en la RCAPA 2015, los que me permitieron corregir algunas imprecisiones. Este trabajo fue financiado con los subsidios CONICET PIP 543 y FONCYT PICT 0950/14. A dos revisores anónimos por los comentarios y por las sugerencias a la primera versión del manuscrito. A S.F. Vizcaíno, como editor de sección de la PE-APA, por su cuidadosa edición, la que me convenció de modificar sustancialmente el manuscrito original y de desdoblarlo en dos trabajos.

\section{BIBLIOGRAFÍA}

Ameghino, F. 1908. Las formaciones sedimentarias de la región litoral de Mar del Plata y Chapalmalán. Anales del Museo Nacional de Buenos Aires 17 (s. 3, 10): 343-428.

Archangelsky, S. 1981. Paleobotánica en Argentina. Publicación Especial de la Asociación Paleontológica Argentina, 25 Aniversario: 3-9.

Asúa, M. de. 2012. Dos siglos y un museo. En: P.E. Penchaszadeh (Ed.), El Museo Argentino de Ciencias Naturales 200 años, Museo Argentino de Ciencias Naturales, Buenos Aires, 175 p.

Ayeza, F. de. 1995. El oratorio del Instituto Saturnino E. Unzué. Serie Comunicaciones Archivo Museo Histórico Municipal "Villa Mitre", Mar del Plata 3: 1-10.

Bartolucci, M. y Pilcic, T. 2009. La ciudad material. En: E. Pastoriza (Directora). Un mar de memoria. Historia e imágenes de Mar del
Plata. Edhasa, Buenos Aires, p. 38-63.

Delgado, S. 2011. La gracia disciplinada: detrás de los muros del Asilo Unzué. Mar del Plata, 1912-1955. Editorial Biblos, Buenos Aires, $218 \mathrm{p}$.

Fernicola, J.C. 2011. Implicancias del conflicto Ameghino-Moreno sobre la colección de mamíferos fósiles realizada por Carlos Ameghino en su primera exploración al río Santa Cruz, Argentina. Revista Museo Argentino de Ciencias Naturales, n.s. 13: 41-57.

Golbert, L. y Roca, E.E. 2010. De la Sociedad de Beneficencia a los Derechos Sociales. Ministerio de Trabajo, Empleo y Seguridad Social, Buenos Aires, $170 \mathrm{p}$.

Hrdlička, A. 1912. Early Man in South America. In collaboration with W. Holmes, B. Willis, F.E. Wright, and C. Fenner. Bureau of American Ethnology, Bulletin 52, 405 p.

Ottone, E.G. 2005. The history of paleobotany in Argentina during the $19^{\text {th }}$ century. En: A.J. Bowden, C.V. Burek y R. Wilding (Eds.), History of Paleobotany: Selected Essays. Special Publications 241, Geological Society, London, p. 281-292.

Pascual, R. 1961. Panorama paleozoológico argentino: Vertebrados. Physis 22: 85-103.

Podgorny, I. 2001. La clasificación de los restos arqueológicos en la Argentina, 1880-1940. Primera parte: La diversidad cultural y el problema de la antigüedad del hombre en el Plata. Saber y Tiempo 3: 5-26.

Podgorny, I. 2009. El sendero del tiempo y de las causas accidentales. Prohistoria Ediciones, Rosario, $331 \mathrm{p}$.

Podgorny, I. y Politis, G. 2000. It is not all roses here: Ales Hrdlička's travelog and his trip to Buenos Aires in 1910. Revista de História da Arte e Arqueología 3: 95-105.

Podgorny, I., Blasco, M.E., Farro, M. y Martínez, A. 2015. Carne da minha carne. 0 museu como identidade do seu fundador nos casos do Museu Nacional de Buenos Aires, o Museu de La Plata e o Museu de Lujan, 1880-1930. En: C. de Mello Vasconcellos, P.P. Funari y A. Carvalho (Eds), Museus e identidades na America Latina. Annablume Editora, Sao Paulo, p. 55-66.

Reig, O.A. 1961. La paleontología de vertebrados en la Argentina. Retrospección y prospectiva. Holmbergia 6: 67-127.

Riccardi, A.C. 1981. Reseña de la historia de la Paleontología de invertebrados en la Argentina. Publicación Especial de la Asociación Paleontológica Argentina, 25 Aniversario: 17-22.

Riccardi, A.C. 2005. La Paleontología de Invertebrados en la Argentina, 1955-2005. Publicación Especial de la Asociación Paleontológica Argentina 10: 53-69.

Riccardi, A.C. 2011. El desarrollo de la Paleontología de invertebrados en el Museo de La Plata. Revista de la Asociación Geológica Argentina 68: 380-391.

Servicio Hidrográfico del Ministerio de Marina, 1916. Provincia de Buenos Aires. Mar del Plata y Bajos de Punta Mogotes. Carta Náutica.

Sociedad de Beneficencia de la Capital, 1913. Memoria del año 1912. La Semana Médica, 409 p.

Somma, A.J. 2013. World Wide Web: http://fotosviejasdemardelplata.blogspot.com.ar/2013/01/asilo-saturnino-unzue_26.html.

Stipanicic, P.N. 1971. Reseña histórica sobre la Paleobotánica en la República Argentina. Ameghiniana 7: 169-175.

Sulek, M. 2010. On the modern meaning of philanthropy. Marty Nonprofit and Voluntary Sector Quarterly 39: 193-212

Thompson, A. y Campetella, M.A. 1994. El "tercer sector" en la historia Argentina. Biblioteca Virtual, Consejo Latinoamericano de Ciencias Sociales, $45 \mathrm{p}$.

Torcelli, A.J. 1936. Correspondencia científica. Obras completas y co- 
rrespondencia científica de Florentino Ameghino. Taller de Impresiones Oficiales del Gobierno de la Provincia de Buenos Aires, La Plata, 23: pp. 237, 243-244, 248, 274.

Vizcaíno, S.F., Bargo, M.S. y Fernicola, J.C. 2013. Expediciones paleontológicas durante los siglos XIX y XX a la Formación Santa Cruz (Mioceno Inferior, Patagonia) y destino de los fósiles. $3^{\circ}$ Congreso Argentino de Historia de la Geología (Salta), Actas: 231246.

Páginas web consultadas:

http://es.wikiarquitectura.com/index.php/Instituto Saturnino Unzue http://www.lacapitalmdp.com/contenidos/fotosfamilia http://www.acciontv.com.ar/soca/unzue/huetel/visita.htm http://fotosviejasdemardelplata.blogspot.com.ar

Doi: 10.5710/PEAPA.26.05.2016.109

Recibido: 20 de Diciembre de 2015

Aceptado: 26 de Mayo de 2016 